\title{
Thrombotic Microangiopathy Revealing Bone Metastases from an Ethmoid Sinus Carcinoma
}

\author{
Mony Chenda Morisse ${ }^{\mathrm{a}} \quad$ Loay Kontar $^{\mathrm{b}}$ Céline Bihan $^{\mathrm{a}} \quad$ Mathieu Boone $^{\mathrm{a}}$ \\ Emma Lachaier $^{a} \quad$ Dimitri Titeca-Beauport $^{\mathrm{b}}$ Julien Maizel ${ }^{\mathrm{b}}$ \\ Bruno Chauffert ${ }^{a}$ \\ ${ }^{a}$ Department of Medical Oncology, University Hospital, Amiens, France; ${ }^{b}$ Medical Intensive \\ Care Unit and INSERM U1088, University Hospital, Amiens, France
}

\section{Keywords}

Thrombotic microangiopathy · Cancer · Ethmoid sinus cancer

\begin{abstract}
Cancer-related thrombotic microangiopathy (TMA) is a rare entity whose clinical and biological characteristics have been described in various tumors. Here we describe the first case of cancer-related TMA revealing diffuse bone metastases from an ethmoid sinus carcinoma.

(C) 2016 The Author(s)

Published by S. Karger AG, Basel

\section{Introduction}

Thrombotic microangiopathy (TMA) may be triggered by numerous diseases, such as scleroderma, antiphospholipid antibody syndrome, pre-eclampsia, radiation nephropathy, renal allograft rejection, hematopoietic stem cell transplantation, infections, drugs and tumors [1]. TMA is evocated in presence of microangiopathic hemolytic anemia and thrombocytopenia, resulting in ischemic complications. Several other symptoms could be associated, such as renal failure, diarrhea classically described in hemolytic uremic syndrome, fever, cardiologic or neurologic manifestations found in thrombotic thrombocytopenic purpura
\end{abstract}




\section{Case Reports in Oncology}

(TTP). In patients with cancer, TMA may be due to chemotherapy, such as gemcitabine, mitomycin or platinum salts, and even to targeted therapies. However, TMA reveals or complicates most often the evolution of cancer itself, defining a special entity called cancer-related TMA, often with a short survival [1]. Cancer-related TMA has never been described as a complication of an ethmoid sinus carcinoma.

\section{Case Report}

A 54-year-old man with no medical history, who was a former carpenter, complained of chronic nasal obstruction. An ethmoid sinus carcinoma was diagnosed by nasal endoscopy in October 2014. The complete surgical resection of a pT3N0M0 tumor was performed. Adjuvant radiotherapy, 60 Gy in 30 fractions, was given on the tumor bed.

A thrombophlebitis of the right saphenous vein required fondaparinux in January 2015. The patient was hospitalized in February 2015 due to deterioration of performance status (ECOG 3), mild confusion and jaundice. Temperature, blood pressure, pulse rate and respiratory rate were normal. Initial laboratory tests showed characteristics of a TMA syndrome (table 1). Myelogram showed an increase of erythroblastic and megakaryocytic lineages without abnormal cells. A low prothrombin time was recorded at $47 \%$ level, while activated partial thromboplastin time and fibrinogen level were normal. Infection check-up was negative (blood culture, virus screening, urine analysis). Hereditary defect in protein C, protein $\mathrm{S}$ or antithrombin III was not found. Research of antiphospholipid antibodies was negative.

Daily blood transfusions were necessary during the first $48 \mathrm{~h}$. Plasmatic exchanges and concomitant systemic corticosteroid treatment were started in the hypothesis of a TTP, but rapidly proved ineffective. This early failure associated with others signs (poor condition, erythromyelemia, disseminated intravascular coagulation signs, moderate thrombocytopenia) suggested a cancer-related TMA with an underlying relapse. Thus, a CT scan was performed and showed diffuse bone metastases. Bone marrow biopsy revealed a CDX2+ and CK20+ adenocarcinoma, compatible with an ethmoid sinus adenocarcinoma.

ADAMTS13 (a disintegrin-like metalloprotease with thrombospondin type I motifs) activity was at $30 \%$ of normal, excluding the diagnosis of TTP.

Cisplatin, infusional 5-fluorouracil and leucovorin, were given every 2 weeks, for 4 months. Laboratory tests progressively turned back to baseline levels (table 1). Blood transfusions were necessary for the first 2 cycles. The patient's performance status recovered to 1 allowing an outpatient management. After 8 cycles, a complete biological response was obtained, while disease was stable on CT scanner re-evaluation (table 1).

TMA relapsed in November 2015, despite maintenance of chemotherapy using carboplatin and 5-fluorouracil ( 4 cycles). No salvage chemotherapy was effective [3 cycles of carboplatin and gemcitabine, then 1 cycle of oxaliplatin, infusional 5 -fluorouracil and leucovorin (FOLFOX)]. The patient died on February 2, 2016.

\section{Discussion}

The first case of cancer-related TMA was reported in 1965 in metastatic gastric cancer [2], followed by several series [3-6]. Nevertheless, cancer-related TMA is uncommon, with about 1 case per year out of 2 million people [7]. Tumors mostly associated with TMA are gastric, breast, lung and prostate cancers, as well as carcinoma of unknown origin [7]. The 


\section{Case Reports in Oncology}

present case is the first to report TMA complicating bone metastases from an ethmoid sinus adenocarcinoma.

In a series of 20 patients with cancer-related TMA compared to 134 adult patients with acquired idiopathic TMA, clinical-biological characteristics associated to a malignant etiology were weight loss, dyspnea, bone pain, disseminated intravascular coagulation, massive erythromyelemia, higher platelet count and less severe renal failure [4]. Other signs of cancer-associated TMA described in a review were older age, male gender, previous diagnosis of cancer, higher LDH level, moderate decrease of ADAMTS 13 activity, and refractoriness to plasmatic exchanges [7]. All of these specific characteristics were also found in a cohort study, in addition to low prothrombin time and normal fibrinogen level [6]. These two features occur in disseminated intravascular coagulation; the normal level of fibrinogen can be explained by presence of inflammation, which increases the fibrinogen level. Our patient presented most of these signs, confirming the specific presentation of cancer-related TMA, especially erythromyelemia and a higher platelet count compared to TTP.

Although the physiopathology of cancer-related TMA remains unclear, several hypotheses have emerged $[8,9]$. First, microangiopathic hemolytic anemia may be the consequence of vascular obstruction by tumor emboli, or intimal proliferation. Second, mucin-producing tumors have been shown to be more often associated with TMA, thus mucin may interact with endothelial function. Finally, direct bone marrow invasion associated with tumoral angiogenesis and myelofibrosis can cause endothelial cell damages [10].

A specific treatment towards the primitive disease is a key point when rapidly begun [9, 11]. Indeed, reviews tend to confirm that plasmatic exchanges do not improve TMA due to systemic malignancies, as in our reported case, whereas chemotherapy was slowly active $[6$, 7]. Unfortunately, TMA relapsed 9 months after initial diagnosis when the tumor became chemoresistant. Due to the initial response to treatment, the survival of our patient (12 months) was relatively long in regard of a series of cancer-related TMA in which the 30-day and 2-year survival rates were 50 and $5 \%$, respectively [4].

Appropriate chemotherapy led to a transient complete response of the biological and clinical signs. Clinical and biological features of TMA in a patient with cancer should alert the clinician on a potential progression of the disease.

\section{Statement of Ethics}

As the patient is dead, informed consent from a next of kin was obtained.

\section{Disclosure Statement}

The authors have no conflicts of interest to declare.

\section{References}

1 Antman KH, Skarin AT, Mayer RJ, et al: Microangiopathic hemolytic anemia and cancer: a review. Medicine (Baltimore) 1979;58:377-384.

2 Brook J, Konwaler BE: Thrombotic thrombocytopenic purpura - association with metastatic gastric carcinoma and a possible auto-immune disorder. Calif Med 1965;102:222-227. 
Morisse et al.: Thrombotic Microangiopathy Revealing Bone Metastases from an Ethmoid Sinus Carcinoma

-3 Francis KK, Kalyanam N, Terrell DR, et al: Disseminated malignancy misdiagnosed as thrombotic thrombocytopenic purpura: a report of 10 patients and a systematic review of published cases. Oncologist 2007;12:11-19.

4 Oberic L, Buffet M, Schwarzinger M, et al: Cancer awareness in atypical thrombotic microangiopathies. Oncologist 2009;14:769-779.

-5 Lechner K, Obermeier HL: Cancer-Related Microangiopathic hemolytic anemia: clinical and laboratory features in 168 reported cases. Medicine (Baltimore) 2012;91:195-205.

6 Ducos G, Mariotte E, Galicier L, et al: Metastatic cancer-related thrombotic microangiopathies: a cohort study. Future Oncol 2014;10:1727-1734.

7 George JN: Systemic malignancies as a cause of unexpected microangiopathic hemolytic anemia and thrombocytopenia. Oncology (Williston Park) 2011;25:908-914.

8 Brain MC, Azzopardi JG, Baker LRI, et al: Microangiopathic haemolytic anaemia and mucin-forming adenocarcinoma. Br J Haematol 1970;18:183-194.

-9 Werner TL, Agarwal N, Carney HM, Rodgers GM: Management of cancer-associated thrombotic microangiopathy: what is the right approach? Am J Hematol 2007;82:295-298.

10 von Bubnoff N, Sandherr M, Schneller F, Peschel C: Thrombotic thrombocytopenic purpura in metastatic carcinoma of the breast. Am J Clin Oncol 2000;23:74-77.

$\$ 11$ Murgo AJ: Thrombotic microangiopathy in the cancer patient including those induced by chemotherapeutic agents. Semin Hematol 1987;24:161-177.

Table 1. Evolution of laboratory studies from initial diagnosis (February 2015) to third-line chemotherapy (December 2015)

\begin{tabular}{llllll}
\hline & $\begin{array}{l}\text { Initial } \\
\text { diagnosis }\end{array}$ & $\begin{array}{l}\text { After } \\
2 \text { cycles }\end{array}$ & $\begin{array}{l}\text { After } \\
8 \text { cycles }\end{array}$ & $\begin{array}{l}\text { First } \\
\text { relapse }\end{array}$ & At third line \\
\hline Hemoglobin, g/dl & 4 & 9.3 & 11 & 5 & 5.8 \\
Reticulocytes, $/ \mathrm{mm}^{3}$ & 146,720 & 160,800 & $\mathrm{x}$ & 80,620 & $\mathrm{x}$ \\
Schistocytes present & yes $(>1 \%)$ & yes $(<0.5 \%)$ & no & no & yes $(>1 \%)$ \\
Platelets, $/ \mathrm{mm}^{3}$ & 83,000 & 249,000 & 232,000 & 46,000 & 12,000 \\
Free bilirubin, $\mu$ mol/l & 31 & 9 & 5 & 18 & 23 \\
Haptoglobin, g/l & $<0.078$ & 1.75 & $\mathrm{x}$ & 0.12 & $<0.078$ \\
LDH, UI/l & 3,388 & 804 & 440 & 1,197 & 2,982 \\
Erythromyelemia, $\%$ & 19 & 2 & no & no & 7 \\
Prothrombin time, $\%$ & 47 & 74 & $\mathrm{x}$ & 67 & 66 \\
Creatinine, $\mu$ mol/l & 83 & 54 & 53 & 94 & 54 \\
\hline
\end{tabular}

$\mathrm{x}=$ Not done. 